\title{
BLM scale fixing in event-shape distributions
}

\author{
Thomas Gehrmann $^{1, \mathrm{a}}$, Niklaus Häfliger ${ }^{1, \mathrm{~b}}$, Pier Francesco Monni ${ }^{1,3,2, \mathrm{c}}$ \\ ${ }^{1}$ Physik-Institut, Universität Zürich, Winterthurerstrasse 190, 8057 Zurich, Switzerland \\ 2 Rudolf Peierls Centre for Theoretical Physics, University of Oxford, 1 Keble Road, Oxford OX1 3NP, UK \\ ${ }^{3}$ Institute for Particle Physics Phenomenology, University of Durham, Durham DH1 3LE, UK
}

Received: 5 February 2014 / Accepted: 9 May 2014 / Published online: 3 June 2014

(C) The Author(s) 2014. This article is published with open access at Springerlink.com

\begin{abstract}
We study the application of the Brodsky-LepageMackenzie (BLM) scale-setting prescription to event-shape distributions in electron-positron collisions. The renormalization scale is set dynamically according to the BLM method. We study NLO predictions and we discuss extensions of the prescription to NNLO.
\end{abstract}

\section{Introduction}

A key problem in making precise predictions in perturbative QCD concerns the choice of the renormalization scale for a process and the theoretical uncertainty associated with it. The dependence of the perturbative result upon such a scale gives an estimate of the size of unknown higher-order perturbative corrections. Since in general only the first few terms of the perturbative series are actually known, it is of primary relevance to figure out how to choose the renormalization scale in order to minimise missing terms. Different possible prescriptions have been proposed in the literature, e.g. fastest apparent convergence [1], principle of minimum sensitivity (PMS) [2] and the Brodsky-LepageMackenzie (BLM) method [3]. Commonly the scale is set to some characteristic scale of the process, which can depend on the observables under consideration. In the present work we apply the BLM method to event-shape distributions in electron-positron collisions. We analyse the standard set of six event-shape observables described in e.g. [4], which have been measured precisely at $e^{+} e^{-}$colliders [5-23]. Perturbative QCD predictions up to NNLO [24,25] and electro-weak

Preprint numbers: ZU-TH 04/14, LPN14-048.

\footnotetext{
a e-mail: thomas.gehrmann@uzh.ch

b e-mail: niklaus@lcc.ch

ce-mail: pier.monni@physics.ox.ac.uk
}

corrections up to next-to-leading order (NLO) [26,27] are available for these observables. Moreover, resummations of Sudakov logarithms have been derived for these observables at different logarithmic accuracies [28-36].

The BLM method was initially formulated for NLO predictions. For differential quantities, the BLM prescription results in a dynamical renormalization scale set on a binby-bin basis. We compare different choices of the renormalization scale and we analyse the extension of the method to NNLO. The resulting predictions are compared to experimental data from the ALEPH collaboration [5,6]. The paper is organised as follows. In Sect. 2 we report the theoretical framework. In Sect. 3 we recall the BLM method and discuss its implementation for event-shape variables and the extension of the approach to NNLO. Numerical results are reported in Sect. 4, while Sect. 5 contains our conclusions.

\section{Event shapes in perturbative QCD}

For the set of event-shape observables analysed here, NNLO predictions were computed in refs. [24,25]. We can express their perturbative expansion in the form

$$
\begin{aligned}
\frac{1}{\sigma} \frac{\mathrm{d} \sigma}{\mathrm{d} y}= & A(y)\left(\frac{\alpha_{s}\left(\mu_{1}\right)}{2 \pi}\right)+B\left(y, \mu_{1}\right)\left(\frac{\alpha_{s}\left(\mu_{2}\right)}{2 \pi}\right)^{2} \\
& +C\left(y, \mu_{1}, \mu_{2}\right)\left(\frac{\alpha_{s}\left(\mu_{3}\right)}{2 \pi}\right)^{3}+\cdots,
\end{aligned}
$$

where $y$ stands for any event-shape variable and the dots indicate missing higher-order corrections of $\mathcal{O}\left(\alpha_{s}^{4}\right)$, with $\sigma$ being the total cross section. The renormalization scales $\mu_{1}$, $\mu_{2}$ and $\mu_{3}$ indicate the scales at which the coupling constant is evaluated in the leading, next-to-leading and next-to-next-toleading order corrections, respectively. The strong coupling in Eq. (1) is commonly evaluated at some renormalization 
scale $\mu$ of the order of the centre-of-mass energy $Q$, i.e. $\mu_{1}=\mu_{2}=\mu_{3}=Q$.

For our analysis it is useful to express explicitly the dependence of the perturbative coefficients in Eq. (1) on the number of active quark flavours $n_{F}$. We thus write

$$
\begin{aligned}
& A(y)=A_{0}(y), \\
& B(y, Q)=B_{0}(y)+B_{1}(y) n_{F}, \\
& C(y, Q, Q)=C_{0}(y)+C_{1}(y) n_{F}+C_{2}(y) n_{F}^{2},
\end{aligned}
$$

where the coefficients $A_{0}, B_{0}, B_{1}, C_{0}, C_{1}$ and $C_{2}$ are evaluated at a fixed renormalization scale $Q$.

\section{The BLM method}

In this section we briefly recall the BLM scale-setting method introduced in [3]. The method gives a simple prescription to set the renormalization scale for a process, in order to improve the convergence of the perturbative expansion. The main idea is to redefine the coupling constant such that all contributions arising from corrections to gauge boson propagators are absorbed into it. In QED, the running of the coupling is exclusively ruled by vacuum polarization insertions in the photon propagator. The latter diagrams are automatically absorbed into the QED coupling constant $\alpha\left(k^{2}\right)$ through the photon wave function renormalization

$\frac{1}{-k^{2}-i 0} \rightarrow \frac{\alpha\left(-k^{2}\right)}{-k^{2}-i 0}$,

which fully defines the QED running coupling. Equation (3) shows that the absorption of vacuum polarization diagrams into the coupling makes the latter run with the virtuality of the virtual photon. This provides us with a prescription to choose the coupling scale when evaluating Feynman diagrams. A direct consequence of this prescription is that each Feynman diagram contributing to a given amplitude will have a different scale at which the coupling must be evaluated. At higher orders, where loop integrals are present, this prescription would make the loop integration quite cumbersome and thus it is not practical. It is then customary to choose a common renormalization scale at which all the couplings $\alpha$ present in the process are evaluated. If one considers using the mean value theorem to evaluate the resulting loop integral, there must be some momentum scale $k^{2}=Q^{* 2}$ which dominates the integral, thus minimizing higher-order corrections. Higher-order corrections would naturally require a different scale, leading to the perturbative expansion

$$
\begin{aligned}
\frac{1}{\sigma} \frac{\mathrm{d} \sigma}{\mathrm{d} y}= & A(y)\left(\frac{\alpha\left(Q^{*}\right)}{2 \pi}\right)+B\left(y, Q^{*}\right)\left(\frac{\alpha\left(Q^{* *}\right)}{2 \pi}\right)^{2} \\
& +C\left(y, Q^{*}, Q^{* *}\right)\left(\frac{\alpha\left(Q^{* * *}\right)}{2 \pi}\right)^{3}+\cdots,
\end{aligned}
$$

for a generic observable $y$.

The BLM method suggests to fix the scales $Q^{*}, Q^{* *}$, $Q^{* * *}, \ldots$ so that to absorb the vacuum polarization contributions into the coupling at each order in perturbation theory. In QED, at low orders we can easily identify those contributions with the $n_{F}$-dependent terms in the perturbative expansion, with $n_{F}$ being the number of active flavours. At higher orders, additional $n_{F}$-dependent terms arise through fermion boxes which are UV finite and thus must not be absorbed into the coupling.

The extension of this prescription to QCD is not trivial, since more diagrams (gluon and ghost loops) contribute to the running of the strong coupling constant. Reference [3] suggests to implement the same prescription used in QED, but with the replacement

$\beta^{\mathrm{QED}} \rightarrow \beta$

where $\beta$ denotes the $\mathrm{QCD} \beta$ function. Once again this amounts to absorbing the vacuum polarization diagrams into the strong coupling constant and to setting the renormalization scales such that $n_{F}$-dependent terms vanish at each order in perturbation theory. This recipe will work unless fermionic box diagrams are present. In this case, it is not possible to disentangle the vacuum polarization contributions from the remaining $n_{F}$ dependent terms and the prescription does not apply. In the process $e^{+} e^{-} \rightarrow$ jets that we want to study, such terms only appear at and beyond NNLO, so the BLM method can be applied at NLO. The prescription outlined in (5) implies that also the $\sim C_{A}$ contributions to vacuum polarization diagrams (due to gluon and ghost loops) are absorbed into the running of $\alpha_{s}$. The final perturbative expansion will be free of vacuum polarization diagrams which are responsible for the leading renormalon growth, i.e. $\sim \alpha_{s}^{n+1} \beta_{0}^{n} n$ ! (see e.g. ref. [38]), so it is expected to have better convergence properties.

The LO BLM renormalization scale $Q^{*}$ can be obtained by cancelling the $n_{F}$ dependence of the NLO coefficient in the expansion

$$
\begin{aligned}
\frac{1}{\sigma} \frac{\mathrm{d} \sigma}{\mathrm{d} y}= & A(y)\left(\frac{\alpha_{s}\left(Q^{*}\right)}{2 \pi}\right)+B\left(y, Q^{*}\right)\left(\frac{\alpha_{s}\left(Q^{* *}\right)}{2 \pi}\right)^{2} \\
& +C\left(y, Q^{*}, Q^{* *}\right)\left(\frac{\alpha_{s}\left(Q^{* * *}\right)}{2 \pi}\right)^{3}+\cdots
\end{aligned}
$$


where the perturbative coefficients read (we set $C_{A}=3$, $C_{F}=4 / 3$ and $T_{F}=1 / 2$ )

$$
\begin{aligned}
& A(y)=A_{0}(y), \\
& B\left(y, Q^{*}\right)=B_{0}(y)+\frac{11}{2} A_{0}(y) \log \frac{Q^{* 2}}{Q^{2}} \\
& +\left(B_{1}(y)-\frac{1}{3} A_{0}(y) \log \frac{Q^{* 2}}{Q^{2}}\right) n_{F}, \\
& C\left(y, Q^{*}, Q^{* *}\right)=C_{0}(y)+\frac{51}{2} A_{0}(y) \ln \frac{Q^{* 2}}{Q^{2}} \\
& -\frac{121}{4} A_{0}(y) \ln ^{2} \frac{Q^{* 2}}{Q^{2}}+\frac{121}{2} A_{0}(y) \ln \frac{Q^{* 2}}{Q^{2}} \ln \frac{Q^{* * 2}}{Q^{2}} \\
& +11 B_{0}(y) \ln \frac{Q^{* * 2}}{Q^{2}} \\
& +n_{F}\left[C_{1}(y)-\frac{19}{6} A_{0}(y) \ln \frac{Q^{* 2}}{Q^{2}}\right. \\
& +\frac{11}{3} A_{0}(y) \ln ^{2} \frac{Q^{* 2}}{Q^{2}}-\frac{22}{3} A_{0}(y) \ln \frac{Q^{* 2}}{Q^{2}} \ln \frac{Q^{* * 2}}{Q^{2}} \\
& \left.+\left(-\frac{2}{3} B_{0}(y)+11 B_{1}(y)\right) \ln \frac{Q^{* * 2}}{Q^{2}}\right] \\
& +n_{F}^{2}\left[C_{2}(y)-\frac{1}{9} A_{0}(y) \ln ^{2} \frac{Q^{* 2}}{Q^{2}}\right. \\
& \left.+\frac{2}{9} A_{0}(y) \ln \frac{Q^{* 2}}{Q^{2}} \ln \frac{Q^{* * 2}}{Q^{2}}-\frac{2}{3} B_{1}(y) \ln \frac{Q^{* * 2}}{Q^{2}}\right] .
\end{aligned}
$$

Using the running coupling expression we find

$Q^{*}=Q \exp \left\{\frac{3 B_{1}(y)}{2 A_{0}(y)}\right\}$,

where $Q$ is the centre-of-mass energy of the process. This amounts to a dynamical scale which is set on a bin-by-bin basis. Considering the expressions reported in Eq. (2), the resulting perturbative series reads

$$
\begin{aligned}
\frac{1}{\sigma} \frac{\mathrm{d} \sigma}{\mathrm{d} y}= & A_{0}(y)\left(\frac{\alpha_{s}\left(Q^{*}\right)}{2 \pi}\right) \\
& +\left(\frac{33}{2} B_{1}(y)+B_{0}(y)\right)\left(\frac{\alpha_{s}\left(Q^{* *}\right)}{2 \pi}\right)^{2}+\cdots
\end{aligned}
$$

The NLO scale $Q^{* *}$ is arbitrary at this order, and it is set by higher-order vacuum polarization diagrams.

\subsection{Extension to higher orders}

In the previous section we recalled the BLM scale-fixing method which led to the perturbative expansion Eq. (9), with the renormalization scale set by Eq. (8). Possible extensions of the BLM prescription to higher orders for inclusive cross sections have been first studied in $[37,40,41]$ and recently a systematic all-order method has been proposed in [42-44]. To extend the method to NNLO differential distributions we have to set the $\mathrm{LO}$ and NLO scales $\left(Q^{*}\right.$ and $\left.Q^{* *}\right)$ in order to absorb all vacuum polarization insertions up to $\mathcal{O}\left(\alpha_{s}^{3}\right)$. This is a non-trivial problem since beyond $\mathrm{NLO} n_{F}$-dependent terms arise also from UV-finite Feynman diagrams. At NNLO such terms can stem either from fermion box insertions (light-bylight diagrams) or from fermion triangles insertions where two fermion legs are cut according to the phase space trigger function. The latter family vanishes in inclusive observables because of Furry's theorem, but they yield a contribution if exclusive phase space cuts are applied. Light-by-light diagrams were found to have a negligible numerical impact [39], and they were discarded in the calculation. Thus they are not included in the event generator EERAD3 that we use to obtain the fixed-order distributions. On the other hand, triangle-like diagrams are numerically sizeable and proportional to $n_{F}$. Their contribution does not take part in the running of the coupling so they must not be absorbed in the scale-fixing procedure.

Looking at Eq. (7) one can see that by plugging the LO expression for the $Q^{*}$ scale into the $C(y)$ coefficient, the $Q^{* *}$ dependence in the $n_{F}^{2}$ contribution gets cancelled. This implies that the only way to absorb the $n_{F}^{n}$ term at $\mathcal{O}\left(\alpha_{s}^{n+1}\right)$ is to modify the LO scale $Q^{*}$ by radiative corrections [3]. It is straightforward to show that this leads to the choice

$$
\begin{aligned}
Q^{*}= & Q \exp \left(\frac{3 B_{1}(y)}{2 A_{0}(y)}\right) \\
& \times\left(1+9 \frac{\alpha_{s}(\mu)}{2 \pi} \beta_{0}\left(\frac{B_{1}^{2}(y)}{A_{0}^{2}(y)}-\frac{C_{2}(y)}{A_{0}(y)}\right)+\cdots\right),
\end{aligned}
$$

where we use the conventions of ref. [33] for the QCD $\beta$ function, i.e. $\beta_{0}=11 / 12 C_{A}-1 / 3 T_{F} n_{F}$. It should be noted that we absorb a contribution proportional to $\beta_{0}$, such that the dependence on the number of active flavours is fully contained in the coefficients of the QCD $\beta$ function. The scale $\mu$ at which the coupling constant in Eq. (10) is evaluated is determined by higher-order corrections to the process and it is arbitrary at this order. It introduces an intrinsic ambiguity similar to the $Q^{* * *}$ scale in the NNLO corrections. The choice (10) guarantees the absence of the leading renormalon $\sim \alpha_{s}^{n+1} \beta_{0}^{n} n$ ! from the perturbative expansion. The NLO scale $Q^{* *}$ can be obtained by absorbing the $\mathcal{O}\left(\alpha_{s}^{3}\right)$ single vacuum polarization insertions (proportional to $n_{F}$ ) into the coupling. It is in general very difficult to single out the contributions of UV-finite diagrams proportional to $n_{F}$ from the remaining vacuum polarization terms and the resulting decomposition is not gauge-invariant. The resulting expression for the $Q^{* *}$ scale reads [37] 
$Q^{* *}=Q \exp \left\{-\frac{3\left(19 B_{1}(y)-2 C_{1}^{\mathrm{VP}}(y)-66 C_{2}(y)\right)}{4\left(2 B_{0}(y)+33 B_{1}(y)\right)}\right\}$,

where $C_{1}^{\mathrm{VP}}(y)$ contains only the gauge-dependent vacuum polarization contributions to the $C_{1}(y)$ coefficient.

In the Feynman gauge, Eq. (11) often leads to very low values of the scale $Q^{* *}$ also in the hard region of the spectrum for almost all of the observables studied here. One may think of replacing $C_{1}^{\mathrm{VP}}(y)$ with $C_{1}(y)$ in Eq. (11) in order to obtain a gauge-invariant prescription. The resulting scales so obtained are still of the order of $\Lambda_{\mathrm{QCD}}$, and this makes the choice in Eq. (11) useless. We thus decide to implement a minimal prescription where we only set the $Q^{*}$ scale to its NLO value [Eq. (10)], while keeping $Q^{* *}=Q^{* * *}=\mu=Q$. We stress that there is no reason why one should set $Q^{* *}=Q^{*}$, since this would introduce spurious $n_{F}$-dependent terms at $\mathcal{O}\left(\alpha_{s}^{3}\right)$.

\section{Numerical results}

In this section we present and discuss the numerical results [45] obtained with the scale-fixing prescriptions discussed above. Distributions are obtained with the generator EERAD3, yielding higher-order coefficients normalised to the Born cross section

$\sigma_{0}=\frac{4 \pi \alpha}{3 s} N e_{q}^{2}$

while the formulas presented in the previous sections are obtained by normalizing the differential cross sections to the total cross section $\sigma$ for $e^{+} e^{-} \rightarrow$ hadrons. One can account for the change of normalization by means of the factor

$$
\begin{aligned}
\frac{\sigma_{0}}{\sigma}=1 & -\frac{3}{2} C_{F}\left(\frac{\alpha_{s}}{2 \pi}\right)+\frac{C_{F}}{8}\left(21 C_{F}+n_{f} T_{F}(44-32 \zeta(3))\right. \\
& \left.+C_{A}(88 \zeta(3)-123)\right)\left(\frac{\alpha_{s}}{2 \pi}\right)^{2}+\mathcal{O}\left(\alpha_{s}^{3}\right) .
\end{aligned}
$$

The running coupling is evaluated using the package RunDec [46]. Figures 1 and 2 show the comparison between the NLO and NNLO distributions evaluated at a fixed renormalization scale $Q$ (red and blue curves, respectively) and two different implementations of the BLM method corresponding to two different choices for the NLO scale $Q^{* *}$. The green curve is obtained by setting $Q^{* *}=Q$ while the orange curve corresponds to $Q^{* *}=Q^{*}$. Experimental data from the ALEPH experiment [5,6] at $Q=M_{Z}$ are also included. The error bands for the standard fixed-order results (red and blue bands) are obtained by varying the renormalization scale initially set to $Q$ by a factor of two in either direction. The bands for the BLM curves are obtained by implementing the latter variation for the $Q^{* *}$ scale around its central value. Such a scale
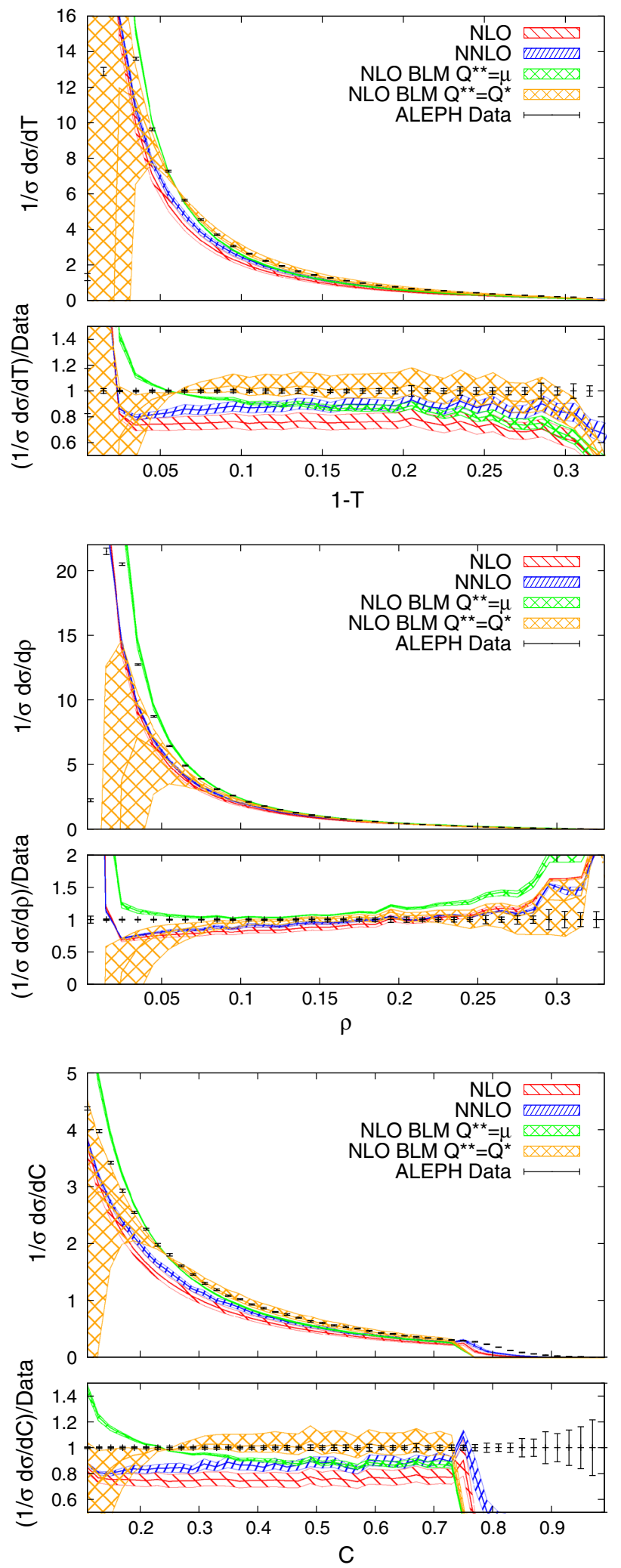

Fig. 1 Distributions for thrust $(T)$, heavy-jet mass $\left(\rho=M_{H}^{2} / Q^{2}\right)$ and C-parameter $(C)$ at $Q=M_{Z}$. The red and blue curves are the fixed-scale NLO and NNLO predictions, respectively. The remaining bands represent the NLO prediction with BLM scale fixing either with $Q^{* *}=Q^{*}$ (orange) or $Q^{* *}=\mu($ green $)$ 

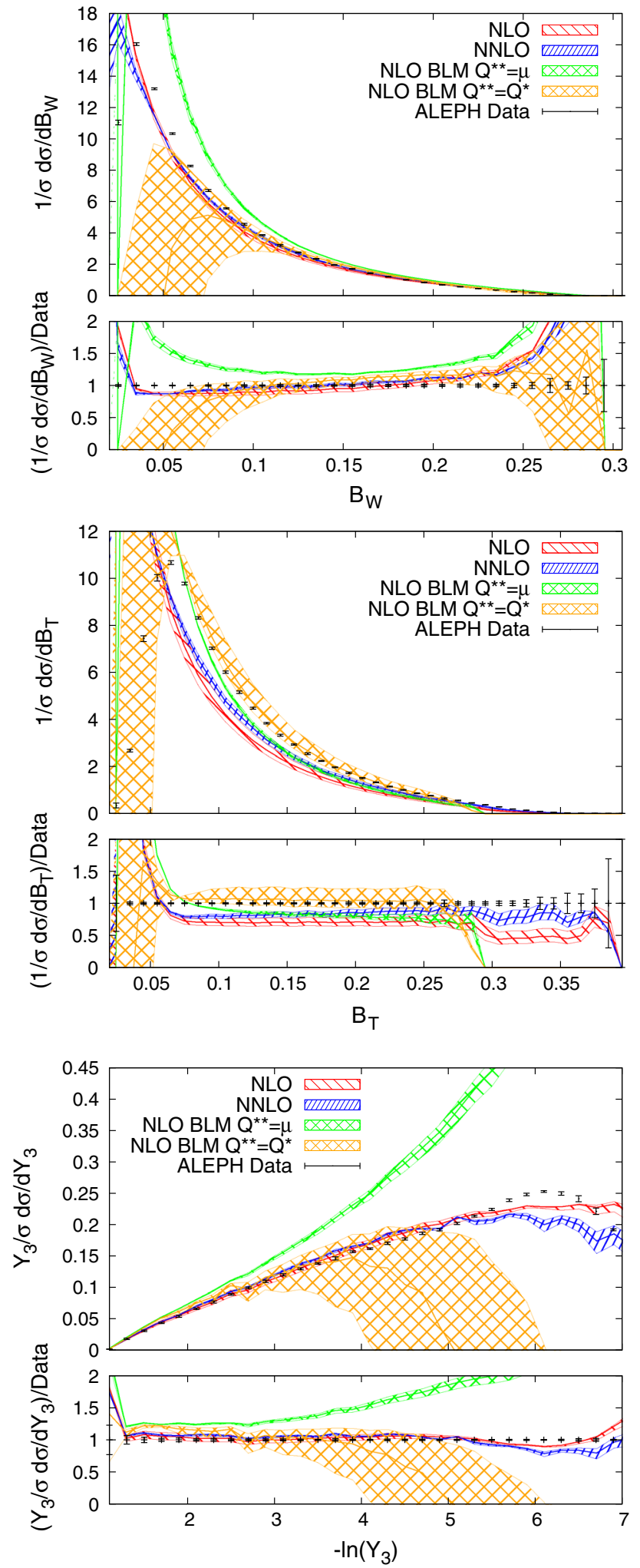

Fig. 2 Distributions for total and wide broadening $\left(B_{W}, B_{T}\right)$ and threejet resolution parameter in the Durham $\left(k_{t}\right)$ algorithm $\left(Y_{3}\right)$ at $Q=M_{Z}$. The red and blue curves are the fixed-scale NLO and NNLO predictions, respectively. The remaining bands represent the NLO prediction with BLM scale fixing either with $Q^{* *}=Q^{*}$ (orange) or $Q^{* *}=\mu$ (green) is ambiguous at this order and its variation gives an estimate of the uncertainty associated with it. We observe that in both cases the BLM prescription gives rise to a harder spectrum for all observables. The choice $Q^{* *}=Q$ (green band) leads to a smaller error band when compared to both the NLO and the NNLO ones. On the other hand, the choice $Q^{* *}=Q^{*}$ (orange band) leads to much larger errors. The distributions obtained with the latter choice are in good agreement with experimental data away from the infrared region. Moreover, when the infrared limit is approached, the fixedorder prediction becomes unreliable and the uncertainty band gets wider. The BLM method cannot be defined in the multijet region beyond the leading-oder kinematical endpoint at which the $A_{0}(y)$ coefficient vanishes, leaving the $Q^{*}$ scale undefined.

Figures 3 and 4 show the comparison between the NNLO distributions obtained with different scale-fixing prescriptions. The blue band corresponds to the standard choice $Q^{*}=Q^{* *}=Q^{* * *}=Q$ for the central scale. Its uncertainty is obtained by varying simultaneously all scales by a factor of two around $Q$. The red band is obtained with $Q^{*}$ set to its NLO value [Eq. (10)] and $Q^{* *}=Q^{* * *}=\mu=Q$, and the uncertainty is obtained by varying the latter three scales by a factor of two in either direction. The orange band represents the NLO result with BLM scale $Q^{* *}=Q^{*}$ discussed above. We observe that the red curve is pushed towards data and the resulting spectrum is harder. The corresponding uncertainties are quite small and comparable to the fixed-scale NNLO ones. It is, however, very difficult to estimate the perturbative uncertainty using the BLM prescription due to the different renormalization scales which enter at different orders. We observe that our prescription gives a good description of experimental data for Thrust, $C$-parameter, heavy-jet mass and total jet broadening while it fails in the case of the widejet broadening and the three-jet resolution parameter.

It is interesting to look at the way the BLM scales behave along the event-shape spectrum. We plot both the LO and the NLO $Q^{*}$ scale in Figs. 5 and 6. The blue curve represents the leading order value, which is independent of any renormalization scale, while its NLO values are spanned by the red band obtained by varying the scale $\mu$ in Eq. (10) by a factor of two around $\mu=Q$. We observe that the BLM method leads to very low renormalization scales, much smaller than the centre-of-mass energy of the process. The smaller renormalization scales, thus the larger coupling, lead to harder distributions as observed above. Moreover, we see that the NLO corrections to the BLM scale $Q^{*}$ are quite moderate for all observables, and that the radiative corrections are always positive. In ref. [37] the BLM method is applied to the perturbative expansion of inclusive physical quantities. The authors exponentiate the NLO corrections to the $Q^{*}$ scale in order to obtain a positive definite quantity. Its expression reads 

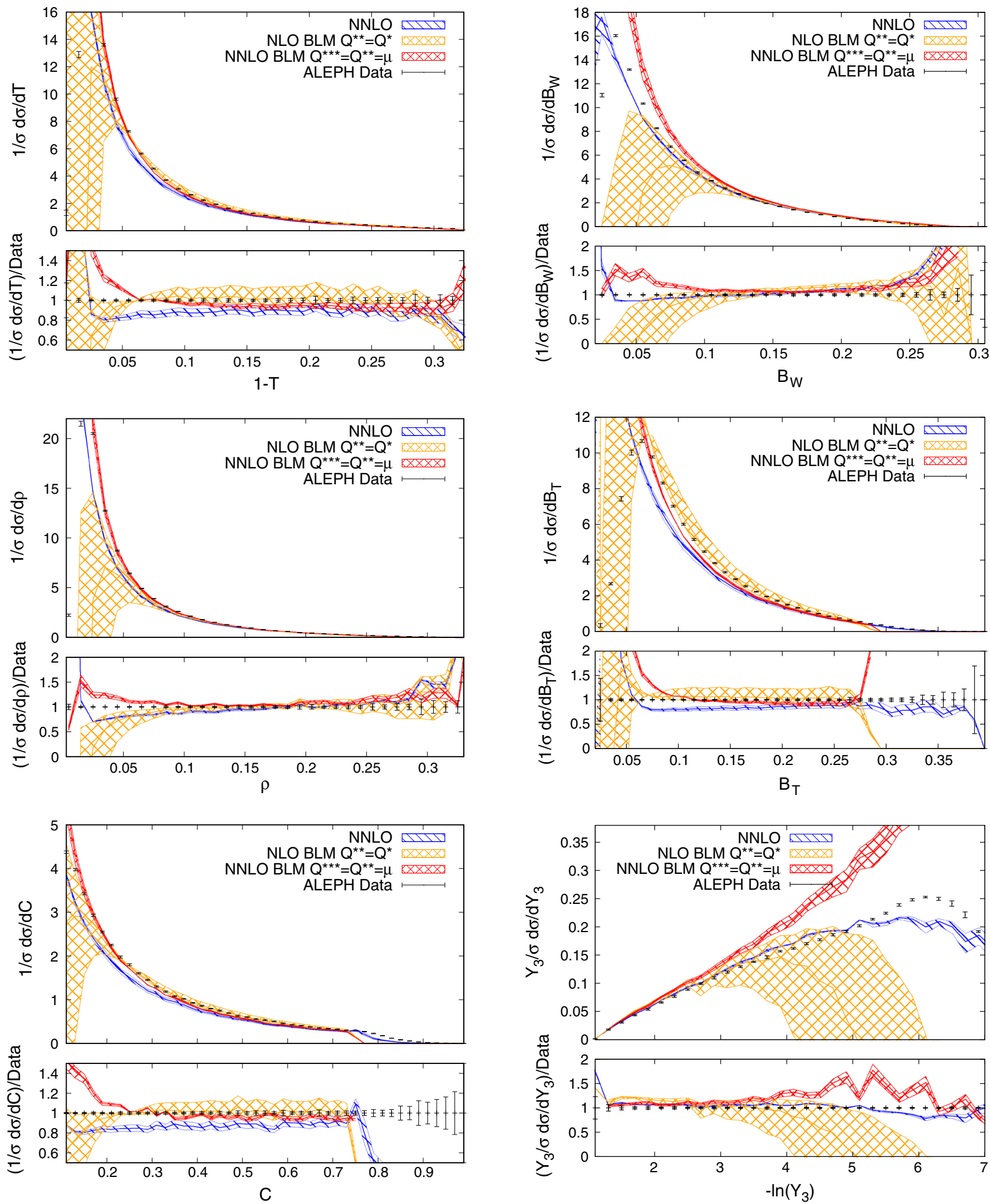

Fig. 3 Distributions for thrust $(T)$, heavy-jet mass $\left(\rho=M_{H}^{2} / Q^{2}\right)$ and $C$-parameter $(C)$ at $Q=M_{Z}$. The red and blue curves represent the NNLO predictions, either with fixed renormalization scale (blue) or with the minimal extension of the BLM method described in the text (red). The orange band represents the NLO prediction with BLM scale fixing with $Q^{* *}=Q^{*}$

Fig. 4 Distributions for wide and total broadening $\left(B_{W}, B_{T}\right)$ and threejet resolution parameter in the Durham algorithm $\left(Y_{3}\right)$ at $Q=M_{Z}$. The red and blue curves represent the NNLO predictions, either with fixed renormalization scale (blue) or with the minimal extension of the BLM method described in the text (red). The orange band represents the NLO prediction with BLM scale fixing with $Q^{* *}=Q^{*}$ 

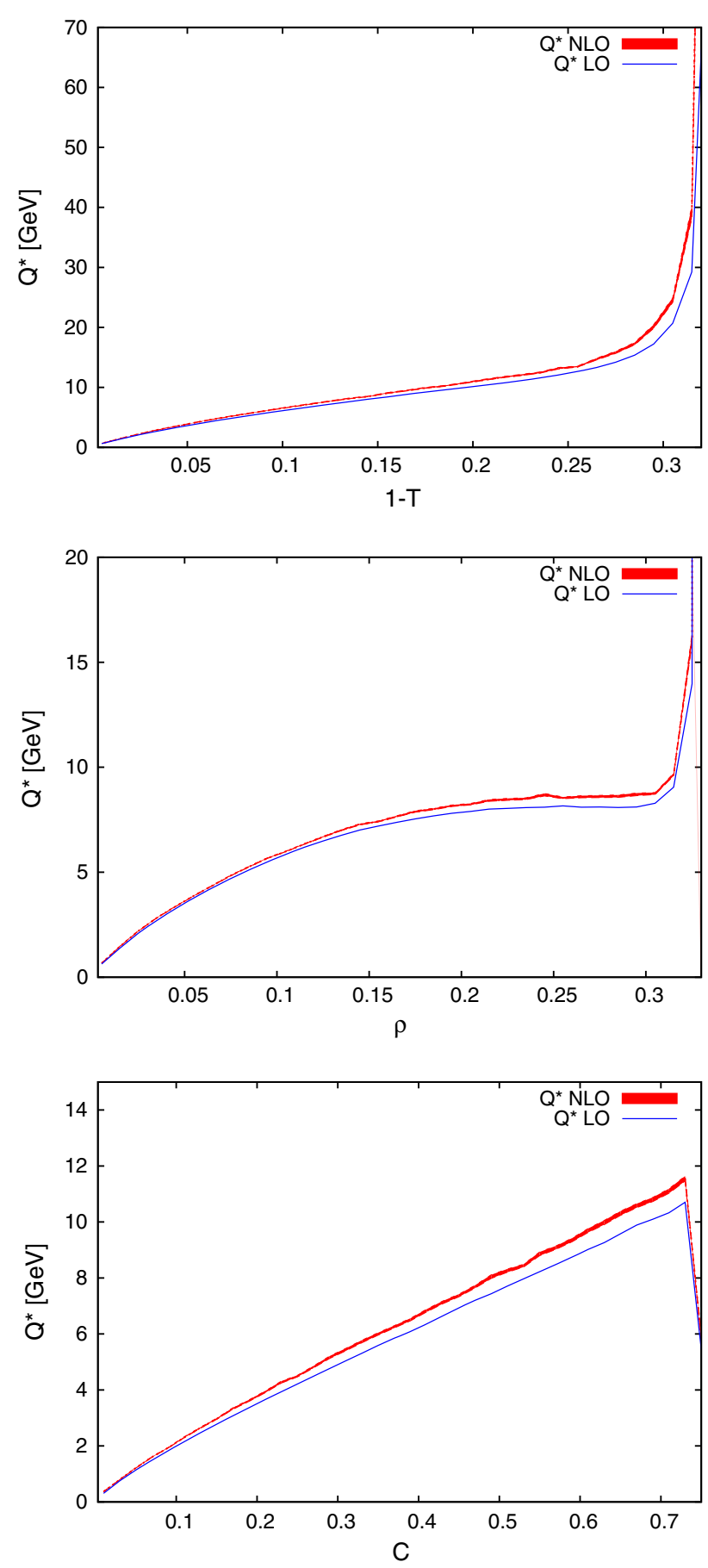

Fig. 5 The LO and NLO BLM scales for thrust $(T)$, heavy-jet mass $\left(\rho=M_{H}^{2} / Q^{2}\right)$ and $C$-parameter $(C)$

$$
\begin{aligned}
Q^{*}= & Q \exp \left(\frac{3 B_{1}(y)}{2 A_{0}(y)}\right. \\
& \left.+9 \frac{\alpha_{s}(\mu)}{2 \pi} \beta_{0}\left(\frac{B_{1}^{2}(y)}{A_{0}^{2}(y)}-\frac{C_{2}(y)}{A_{0}(y)}\right)+\cdots\right) .
\end{aligned}
$$

Nevertheless, in our case this is not a good approximation of the correct $Q^{*}$ scale due to the moderately large NLO
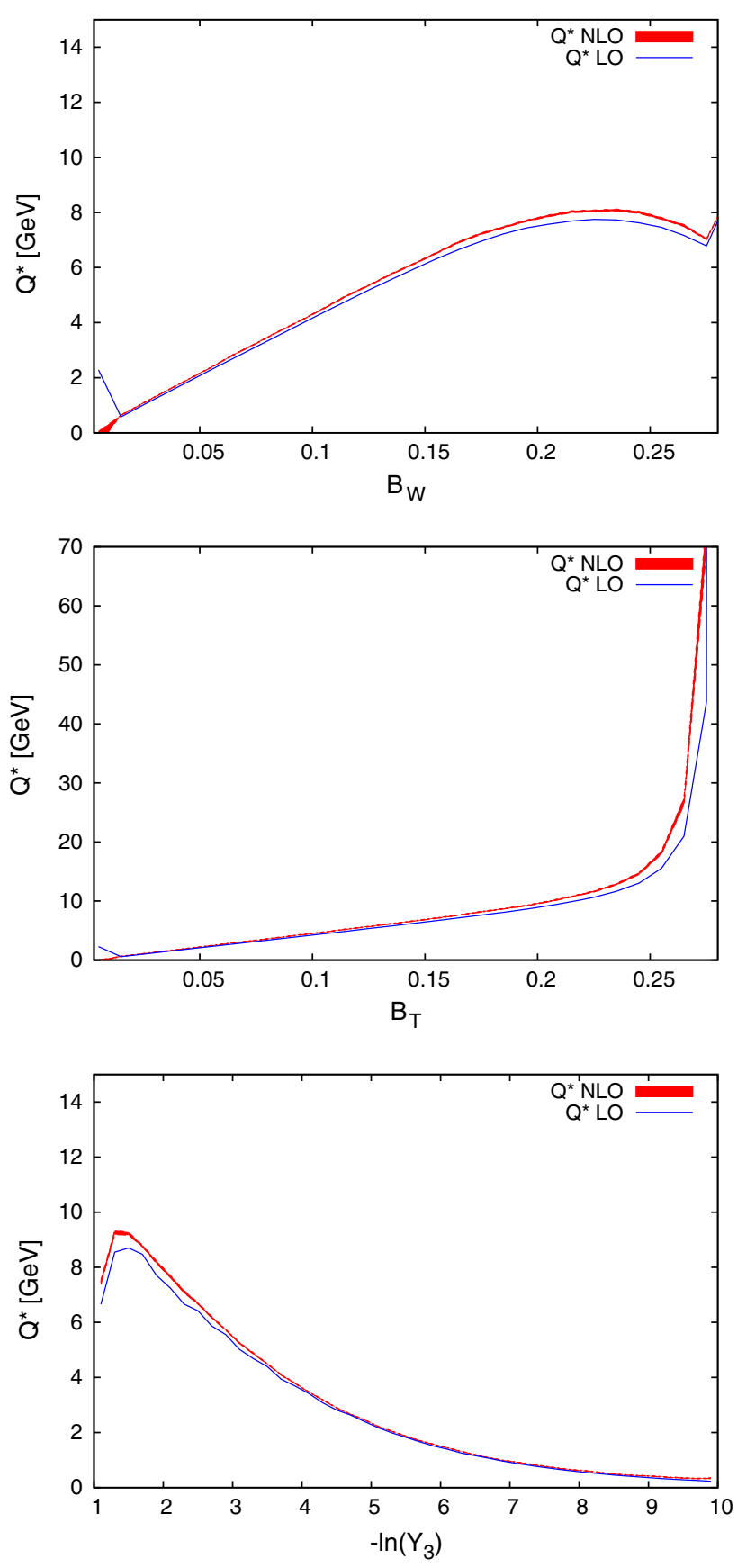

Fig. 6 The LO and NLO BLM scales for wide and total broadening $\left(B_{W}, B_{T}\right)$, and three-jet resolution parameter in the Durham algorithm $\left(Y_{3}\right)$

corrections. In practice, the two results Eq. (10) and Eq. (14) lead to very different numerical values for $Q^{*}$.

\section{Conclusions}

In this paper we studied the impact of the BLM scale-setting method on event-shape distributions in electron-positron col- 
lisions. We found good agreement between the NLO prediction with $Q^{* *}=Q^{*}$ and ALEPH experimental data at the $Z$-boson peak. The theoretical uncertainties associated with the latter predictions are larger than the ones associated with the fixed-scale distributions.

We also analysed the extension of the prescription beyond NLO and found that the scale $Q^{* *}$ cannot be defined in a gauge-invariant manner for the differential cross sections studied here. This is due to the presence of UV-finite and $n_{F}$-dependent terms already at $\mathcal{O}\left(\alpha_{s}^{3}\right)$. Hence, the prescription suggested in [37] is in general not well-defined for noninclusive quantities for which such terms are present. Moreover, the resulting NLO scale $Q^{* *}$ assumes very low values and often probes the non-perturbative regime of the strong coupling constant. We therefore implement a minimal prescription in which we set $Q^{*}$ to its NLO value (10) while setting $Q^{* *}=Q^{* * *}=\mu=Q$. This prescription ensures the absence of the leading renormalon $\sim \alpha_{s}^{n+1} \beta_{0}^{n} n$ ! ambiguity (up to higher-order corrections) at a given order in the perturbative expansion. The agreement with experimental data away from the Sudakov region is remarkable for all observables but the wide-jet broadening and the three-jet resolution parameter. We observe a scale uncertainty of roughly the same size of the fixed-scale NNLO one, and the resulting distributions are harder. The renormalization scales obtained with the BLM method are quite small (of the order of 10$20 \mathrm{GeV}$ in the hard region of the spectrum) and radiative corrections to their value are moderate.

Acknowledgments This work was supported by the Swiss National Science Foundation (SNF) under Grant 200020-138206 and the European Commission through the LHCPhenoNet network under contract PITN-GA-2010-264564.

Open Access This article is distributed under the terms of the Creative Commons Attribution License which permits any use, distribution, and reproduction in any medium, provided the original author(s) and the source are credited.

Funded by $\mathrm{SCOAP}^{3}$ / License Version CC BY 4.0.

\section{References}

1. G. Grunberg, Phys. Lett. B 95, 70 (1980). [Erratum-ibid. B 110 (1982) 501]

2. P.M. Stevenson, Phys. Lett. B 100, 61 (1981)

3. S.J. Brodsky, G.P. Lepage, P.B. Mackenzie, Phys. Rev. D 28, 228 (1983)

4. R.W.L. Jones, M. Ford, G.P. Salam, H. Stenzel, D. Wicke, JHEP 0312, 007 (2003). [hep-ph/0312016]

5. D. Buskulic et al., ALEPH collaboration. Z. Phys. C 73, 409 (1997)

6. A. Heister et al., ALEPH collaboration. Eur. Phys. J. C 35, 457 (2004)

7. P.D. Acton et al., OPAL collaboration. Z. Phys. C 59, 1 (1993)

8. G. Alexander et al., OPAL collaboration. Z. Phys. C 72, 191 (1996)

9. K. Ackerstaff et al., OPAL collaboration. Z. Phys. C 75, 193 (1997)

10. G. Abbiendi et al., OPAL collaboration. Eur. Phys. J. C 16, 185 (2000). [hep-ex/0002012]
11. G. Abbiendi et al., OPAL collaboration. Eur. Phys. J. C 40, 287 (2005). [hep-ex/0503051]

12. G. Abbiendi et al., OPAL collaboration. Eur. Phys. J. C 53, 21 (2008)

13. M. Acciarri et al., L3 collaboration. Phys. Lett. B 371, 137 (1996)

14. M. Acciarri et al., L3 collaboration. Phys. Lett. B 404, 390 (1997)

15. M. Acciarri et al., L3 collaboration. Phys. Lett. B 444, 569 (1998)

16. P. Achard et al., L3 collaboration. Phys. Lett. B 536, 217 (2002). [hep-ex/0206052]

17. P. Achard et al., L3 collaboration. Phys. Rept. 399, 71 (2004). [hep-ex/0406049]

18. P. Abreu et al., DELPHI collaboration. Phys. Lett. B 456, 322 (1999)

19. J. Abdallah et al., DELPHI collaboration. Eur. Phys. J. C 29, 285 (2003). [hep-ex/0307048]

20. J. Abdallah et al., DELPHI collaboration. Eur. Phys. J. C 37, 1 (2004). [hep-ex/0406011]

21. K. Abe et al., SLD collaboration. Phys. Rev. D 51, 962 (1995). [hep-ex/9501003]

22. P. Pfeifenschneider et al., JADE collaboration. Eur. Phys. J. C 17, 19 (2000). [hep-ex/0001055]

23. W. Braunschweig et al., TASSO collaboration. Z. Phys. C 47, 187 (1990)

24. A. Gehrmann-De Ridder, T. Gehrmann, E.W.N. Glover, G. Heinrich, JHEP 0712, 094 (2007). [arXiv:0711.4711]

25. S. Weinzierl, JHEP 0906, 041 (2009). [arXiv:0904.1077]

26. A. Denner, S. Dittmaier, T. Gehrmann, C. Kurz, Phys. Lett. B 679, 219 (2009). [arXiv:0906.0372]

27. A. Denner, S. Dittmaier, T. Gehrmann, C. Kurz, Nucl. Phys. B 836, 37 (2010). [arXiv:1003.0986]

28. S. Catani, L. Trentadue, G. Turnock, B.R. Webber, Nucl. Phys. B 407, 3 (1993)

29. S. Catani, B.R. Webber, Phys. Lett. B 377, 427 (1998). [hep-ph/9801350]

30. Y.L. Dokshitzer, A. Lucenti, G. Marchesini, G.P. Salam, JHEP 011, 9801 (1998). [hep-ph/9801324]

31. A. Banfi, G.P. Salam, G. Zanderighi, JHEP 0201, 018 (2002). [hep-ph/0112156]

32. T. Becher, M.D. Schwartz, JHEP 0807, 034 (2008). [arXiv:0803.0342]

33. P.F. Monni, T. Gehrmann, G. Luisoni, JHEP 1108, 010 (2011). [arXiv: 1105.4560]

34. Y.T. Chien, M.D. Schwartz, JHEP 1008, 058 (2010). [arXiv:1005.1644]

35. T. Becher, G. Bell, JHEP 1211, 126 (2012). [arXiv:1210.0580]

36. T. Becher, G. Bell, M. Neubert, Phys. Lett. B 704, 276 (2011). [arXiv: 1104.4108]

37. S.J. Brodsky, H.J. Lu, Phys. Rev. D 51, 3652 (1995). [hep-ph/9405218]

38. M. Beneke, Phys. Rep. 317, 1 (1999). [hep-ph/9807443]

39. J.J. van der Bij, E.W.N. Glover, Nucl. Phys. B 313, 237 (1989)

40. G. Grunberg, A.L. Kataev, Phys. Lett. B 279, 352 (1992)

41. S.V. Mikhailov, JHEP 0706, 009 (2007). [hep-ph/0411397]

42. S.J. Brodsky, X.G. Wu, Phys. Rev. D 86, 054018 (2012). [arXiv:1208.0700][hep-ph]

43. M. Mojaza, S.J. Brodsky, X.G. Wu, Phys. Rev. Lett. 110, 192001 (2013). [arXiv: 1212.0049][hep-ph]

44. S.J. Brodsky, M. Mojaza, X.G. Wu, Phys. Rev. D 89, 014027 (2014). [arXiv:1304.4631][hep-ph]

45. N. Häfliger, B.Sc. Thesis, University of Zürich (2012)

46. K.G. Chetyrkin, J.H. Kuhn, M. Steinhauser, Comput. Phys. Commun. 133, 43 (2000). [hep-ph/0004189] 